\title{
Electronic case reporting of STIs: Are non-existent codes the reason for missing information?
}

\author{
Crystal Snare ${ }^{\star 1}$, Rita Altamore ${ }^{1}$, Julie Simon ${ }^{1}$, Kim Peifer $^{1}$, Mary Stark $^{2}$ and \\ Bryant Karras ${ }^{1}$
}

${ }^{1}$ DSHS - STD - Infectious disease assessment unit, Washington State Department of Health, Olympia, WA, USA; ${ }^{2}$ Planned

Parenthood of the Great Northwest and the Hawaiian Islands, Boise, ID, USA

\section{Objective}

Previous research identified data gaps between traditional paperbased STI notifiable condition reporting and pilot electronic initial case reporting (eICR) relying on Continuity of Care Documents (CCDs) exported from our clinical partner's electronic health record (EHR) software. ${ }^{1}$ Structured data capture is needed for automatic processing of eICR data imported into public health repositories and surveillance systems, similar to electronic laboratory reporting (ELR). Coding data gaps (between paper and electronic case reports) using standardized vocabularies will allow integration of additional questions into EHR or other data collection systems and may allow creation of standard Clinical Data Architecture (CDA) templates, Logical Observation Identifiers Names and Codes (LOINC) panels, or Fast Healthcare Interoperability Resources (FHIR) resources. Furthermore, identifying data gaps can inform improvements to other standards including nationwide standardization efforts for notifiable conditions.

\section{Introduction}

Under the CDC STD Surveillance Network (SSuN) Part B grant, WA DOH is testing eICR of sexually transmitted infections (STI) with a clinical partner. Existing standard vocabulary codes were identified to represent previously-identified information gaps, or the need for new codes or concepts was identified.

\section{Methods}

In prior work, CCDs were securely received from our clinical partner and then analyzed for gaps compared to the existing paper-based STI case reporting form. ${ }^{1}$ Now, codes associated with gap concepts were identified in standard vocabularies such as LOINC and SNOMED CT. Standards were searched using online browsers offered by the standards development organizations (SDO) to identify potential codes, which were reviewed with public health epidemiologists and clinicians. Gaps were listed, and wording and intent was compared to standard codes including accessory information found in the SDO browsers and a final table of recommended codes was produced.

\section{Results}

Acceptable congruity between currently used case reporting questions and coded vocabularies was found for the majority of data gaps previously identified. Where data need was incongruous with standard coded vocabularies, new codes or concepts could be proposed to the SDO.

Pregnancy status is often missing from CDA documents but is well-conceptualized in both LOINC and SNOMED CT systems under several codes, including any current laboratory tests for pregnancy. HIV status is similarly well-conceptualized in both LOINC and SNOMED CT both as a problem list item well as thru a variety of laboratory tests. However, problem list EHR models lack standard inclusion of date of last HIV test or dates of current pregnancy as an associated coded data element which is desirable for public health.

Information about the case patient's sexual partners, need for partner STI treatment, and partner treatment completed is lacking in standard CCD documents. ${ }^{1}$ Number of current sexual partners [requiring treatment] - has a SNOMED-CT code, but lacks a match in LOINC coding system. This gap identified the need for exploration of how information about sexual partners can best be represented in interoperability artifacts, including the most useful division of information content between the information model and the standard terminology. Needed concept could resemble 'record target' found in HL7 version 3.0 and would allow data to be provided without specifying additional codes. Exploration of information model options or new codes is recommended.

Many codes are possible for site of infection and specific symptoms but the overall concepts of 'symptomatic infection' and 'site of infection' as coded elements would need to be added.

\section{Conclusions}

This coded element gap analysis found that most information requested in an STI case report can be found in a CCD. Gaps can be addressed by using existing standard terms except for concepts about sexual partners that might be better addressed by exploring the information model rather than through the addition of standard codes. Standards use will facilitate complete case reporting using CDA or FIHR, for example, within a 'blue button' or other system with functionality for exchanging additional information about notifiable condition case patients. Collaboration with clinicians, public health practitioners, informaticians, and EHR vendors in will help determine how these concepts might best be modeled. Understanding data gaps involves working closely with a broad range of stakeholders, to understand why gaps exist and how well proposed solutions will meet stakeholder needs.

\section{Keywords}

electronic case reporting; Public Health Informatics; electronic health records; data sharing; sexually transmitted diseases

\section{Acknowledgments}

Funding for this project in part provided by the U.S. Centers for Disease Control and Prevention, STD Surveillance Network, PS13-1306

Washington DOH SSuN Part B team, including Teal Bell, Tom Jaenicke, and Travis Kushner

Planned Parenthood of the Great Northwest and Hawaiian Islands, especially Dr. Laurel Kuehl

\section{References}

1. Peifer, K., Stark, M., Altamore, R., \& Simon, J. Electronic Case Reporting of STIs: Assessing EHR Generated CCDs. OJPHI. 2017 September; 9(1). doi:http://dx.doi.org/10.5210/ojphi.v9i1.7614

\section{*Crystal Snare}

E-mail: crystal.snare@doh.wa.gov 